\title{
Association of Posterior Reversible Encephalopathy Syndrome and Transient Apical Ballooning Syndrome (Takotsubo): First Case Report of a Man and Review of the Literature
}

\author{
Stephan Grimaldi ${ }^{\mathrm{a}} \quad$ Emilie Doche $^{\mathrm{a}}$ Caroline Rey $^{\mathrm{a}}$ Nadia Laksiri $^{\mathrm{a}}$ \\ Salah Boussen ${ }^{b}$ Jacques Quilicic ${ }^{c}$ Emmanuelle Robinet ${ }^{a}$ \\ Fabien Devemy ${ }^{d}$ Jean Pelletier ${ }^{a}$ \\ ${ }^{a}$ Aix Marseille Université, APHM, Hôpital de la Timone, Pôle de Neurosciences Cliniques, \\ Service de Neurologie et Unité NeuroVasculaire, Marseille, France; ${ }^{b}$ Aix Marseille \\ Université, APHM, Hôpital de la Timone, Pôle Anesthésie Réanimation, Service \\ d'Anesthésie Réanimation 1, Marseille, France; ${ }^{c}$ Aix Marseille Université, APHM, Hôpital de \\ la Timone, Pôle Cardiovasculaire et Thoracique, Service de Cardiologie et Maladies \\ Coronaires, Marseille, France; ${ }^{d}$ Centre Hospitalier Alpes du Sud, Service de Cardiologie, \\ Gap, France
}

\section{Keywords}

Posterior reversible encephalopathy syndrome $\cdot$ Takotsubo cardiomyopathy $\cdot$ Catecholamines

\begin{abstract}
Introduction: An association of posterior reversible encephalopathy syndrome (PRES) and takotsubo is rare. We present the first case of a male patient. Case Report: A 69-year-old man presented to the hospital in a persistent comatose state following a generalized tonicclonic seizure with high blood pressure. The electrocardiogram revealed transient left bundle branch block. Troponin and BNP were elevated. Cardiac ultrasound showed large apical akinesia with altered left ventricular ejection fraction, and the left ventriculogram showed characteristic regional wall motion abnormalities involving the mid and apical segments. Brain MRI showed bilateral, cortical, and subcortical vasogenic edema predominant in the
\end{abstract}




\section{Case Reports in Neurology}

posterior right hemisphere. The lumbar puncture and cerebral angiography were normal. Paraclinical abnormalities were reversible within 2 weeks with a clinical recovery in 3 months, confirming the takotsubo and the PRES diagnoses. Discussion: Several theories hypothesize the underlying pathophysiology of takotsubo or PRES. Circulating catecholamines are up to 3 times higher in patients with takotsubo causing impaired microcirculation and apical hypokinesia. An association of both takotsubo and asthma crisis and PRES and asthma crisis underlines the role of catecholamines in the occurrence of these disorders. Conclusion: Early recognition of this rare association, in which heart and neurological damage may require rapid intensive care support, is needed.

(C) 2017 The Author(s)

Published by S. Karger AG, Basel

\section{Introduction}

Only one study reported the association of posterior reversible encephalopathy syndrome (PRES) and transient apical ballooning syndrome (takotsubo cardiomyopathy) and described a series of 6 women in the United States [1]. Five other cases of women were reported separately [2-6]. We present here the first case of a male patient with this rare association.

\section{Case Report}

A 69-year-old Caucasian man was admitted to intensive care unit because of a persistent comatose state (Glasgow Coma Scale scored at 6) following a generalized tonic-clonic seizure requiring intubation. His high blood pressure $(>190 / 110 \mathrm{~mm} \mathrm{Hg})$ required intravenous infusion of nicardipine. Brain CT scan and MRI (with DWI, FLAIR, T2, and T2*) showed no abnormalities. He had a past medial history of oral cancer treated in 2007 with surgery and radiotherapy. He had a comorbid condition of obesity and dyslipidemia. Electrocardiogram (ECG) revealed transient left bundle branch block (Fig. 1a) which normalized a few hours later. Troponin and BNP were elevated to $35 \mathrm{ng} / \mathrm{mL}(n<0.05)$ and $600 \mathrm{pg} / \mathrm{mL}(n<100)$, respectively. Cardiac ultrasound showed large apical akinesia with altered left ventricular ejection fraction (LVEF). Angiography showed no occlusive lesion or stenosis, and left ventriculogram showed regional wall motion abnormalities involving the mid and apical segments, characteristic of takotsubo cardiomyopathy (Fig. 1b).

After 3 days, the patient's consciousness improved. However, he continued to have persistent left-sided hemiparesis predominantly in the upper limb. Electroencephalogram showed slight slowing of cerebral activity in the right hemisphere without paroxysmal activity. The CSF analysis was normal (biochemistry, bacteriology, virology, pathology). A second brain MRI was performed on day 3 (Fig. 1c) and displayed bilateral, cortical, and subcortical vasogenic edema with a right posterior predominance, suggesting the diagnosis of PRES. A cerebral angiography performed on day 12 showed no abnormalities and in particular no stenosis that could have been in favor of a reversible cerebral vasoconstriction syndrome.

Troponin, BNP, LVEF, and cardiac kinetics normalized a few days later as would be expected in transient apical ballooning syndrome. A brain MRI obtained 15 days after admission (Fig. 1d) showed complete resolution of the vasogenic edema confirming the diagnosis of PRES. The patient recovered fully within 3 months. 
In the history there was no record of a stressful physical or emotional event preceding the onset of the patient's symptoms. Biological samples and abdomen/pelvic CT scan found no evidence of thyroid dysfunction or pheochromocytoma.

\section{Discussion}

Takotsubo cardiomyopathy refers to transient apical dyskinesia with normal coronary angiogram mimicking acute coronary syndrome. It presents mainly in middle-aged and elderly women and is often preceded by physical and/or emotional stress. ECG displays ST segment elevation and T wave abnormalities. Troponin may be elevated. Cardiac ultrasound shows left ventricular dysfunction with apical akinesia or dyskinesia and hypercontractility of basal segments, resulting in the expression "octopus trap" (takotsubo in Japanese) morphology of the ventricles. Complications can be severe with acute pulmonary edema or death. Takotsubo cardiomyopathy/physiopathology is not fully understood yet. Several pathological mechanisms have been proposed, including coronary artery vasospasm, coronary microcirculation dysfunction, obstruction of the left ventricular outflow tract, and catecholamine overload [7]. Blood catecholamine levels are up to 3 times higher in takotsubo patients compared to patients with acute coronary syndrome. Thus, catecholamine overload might play a central role in the development of this disorder. Published data suggest that elevated plasma catecholamine levels seen in stress cardiomyopathy patients could be particularly relevant and result in catecholamine-related toxic effects [8]. Stress cardiomyopathy is characterized by morphological alterations that are similar to those following catecholamine cardiotoxic effects reported previously [9] and is thought to be due to changes in microcirculation. Cardiac SPECT analysis shows apical ischemia despite normal coronary arteries [10]. Clinical parameters resolve completely in 1-3 weeks.

PRES is characterized by neurological symptoms including headache, altered mental status, visual disturbances, and seizures in conjunction with reversal of findings on imaging. Brain MRI usually shows subcortical white matter vasogenic edema typically in the parietal and occipital lobes and less frequently in the frontal lobes, cerebellum, basal ganglia, or brainstem [11]. The incidence is moderately higher in females with a mean age of 44 years. Comorbid conditions are hypertension, malignancy, autoimmune diseases, kidney diseases, dialysis-dependent renal insufficiency, and organ transplantation. PRES physiopathology is not yet fully understood as well. Several theories hypothesize on the underlying pathophysiology of PRES: the "vasogenic" theory (with increased systemic blood pressure), the "cytotoxic" theory (toxins responsible for endothelial dysfunction), the "immunogenic" theory (Tcell activation increasing endothelial permeability), and the "neuropeptide" theory (release of vasoconstrictors leading to vasospasm) [12].

Takotsubo cardiomyopathy is commonly described in patients with subarachnoid hemorrhage, status epilepticus, ischemic stroke, craniocerebral trauma, encephalitis, myelitis, Guillain-Barré syndrome, following neurosurgical procedures, acute hydrocephalus, but an association of PRES and takotsubo has rarely been reported. All these situations might be considered as stressful events for the organism and cellular activities. They all might cause an acute dysregulation of cerebral vascular tone because of a sympathetic hyperactivity, endothelial dysfunction, and oxidative stress, but the precise mechanisms remain unknown. To this day, we do not know for certain that catecholamine overload is involved in all the situations associating takotsubo cardiomyopathy and cerebral injuries, but all the data we have reported suggest that it could be particularly relevant. A "catecholaminergic storm" 


\section{Case Reports in Neurology}

Grimaldi et al.: Association of Posterior Reversible Encephalopathy Syndrome and Transient Apical Ballooning Syndrome (Takotsubo)

might lead to myocardial apical hypokynesia (high levels of circulating epinephrine trigger a switch in intracellular signal trafficking, from $G_{s}$ protein to $G_{i}$ protein signaling through the $\beta_{2}$ receptors [13]), blood pressure elevation, and alteration of cerebral microcirculation. Moreover, the association of both takotsubo and an asthma crisis [14] and of PRES and an asthma crisis [15] also underlines the hypothetical role of catecholamines in the occurrence of these disorders.

The literature review found 5 case reports of women [2-6]. Six others cases of women with PRES were found in a series of 224 patients with takotsubo [1]. To our knowledge, this is the first case of a male patient presenting with PRES and takotsubo. The reason for the much more common occurrence in women (and especially in postmenopausal women) has not yet been explained. One hypothesis is that sex hormones may exert important influences on the sympathetic neurohormonal axis and on vasoreactivity. Another one is that women seem to be more vulnerable to sympathetically mediated myocardial stunning and to postmenopausal alteration of endothelial function in response to reduced estrogen levels [7].

\section{Conclusion}

This case highlights the need for early recognition of this rare association, in which heart and neurological damage may require rapid intensive care support. Catecholamine overload seems to play a major role in this phenomenon.

\section{Statement of Ethics}

The authors have no ethical conflicts to disclose.

\section{Disclosure Statement}

The authors declare no financial conflict of interest.

\section{Funding Sources}

No funding sources.

\section{References}

-1 Summers MR, Madhavan M, Chokka RG, Rabinstein AA, Prasad A: Coincidence of apical ballooning syndrome (tako-tsubo/stress cardiomyopathy) and posterior reversible encephalopathy syndrome: potential common substrate and pathophysiology? J Card Fail 2012;18:120-125.

-2 Tajima Y, Matsumoto A: Reversible posterior leukoencephalopathy syndrome in p-ANCA-associated vasculitis. Intern Med 2006;45:1169-1171.

3 Papanikolaou J, Tsirantonaki M, Koukoulitsios G, Papageorgiou D, Mandila C, Karakitsos D, Karabinis A: Reversible posterior leukoencephalopathy syndrome and takotsubo cardiomyopathy: the role of echocardiographic monitoring in the ICU. Hellenic J Cardiol 2009;50:436-438.

-4 Awatsu Y, Morita M, Nakano I: A case of posterior reversible encephalopathy syndrome complicated by takotsubo cardiomyopathy and hypercatecholaminemia. Auton Neurosci 2007;135:144. 


\section{Case Reports in Neurology}

5 Banuelos PA, Temes R, Lee VH: Neurogenic stunned myocardium associated with reversible posterior leukoencephalopathy syndrome. Neurocrit Care 2008;9:108-111.

-6 Fugate JE, Wijdicks EF, Kumar G, Rabinstein AA: One thing leads to another: GBS complicated by PRES and Takotsubo cardiomyopathy. Neurocrit Care 2009;11:395.

7 Gianni M, Dentali F, Grandi AM, Sumner G, Hiralal R, Lonn E: Apical ballooning syndrome or takotsubo cardiomyopathy: a systematic review. Eur Heart J 2006;27:1523-1529.

-8 Wittstein IS, Thiemann DR, Lima JA, Baughman KL, Schulman SP, Gerstenblith G, Wu KC, Rade JJ, Bivalacqua TJ, Champion HC: Neurohumoral features of myocardial stunning due to sudden emotional stress. N Engl J Med 2005;352:539-548.

-9 Yamanaka 0, Fujiwara Y, Nakamura T, Ohno A, Endo Y, Yoshimi K, Miura K, Yamaguchi H: "Myocardial stunning"-like phenomenon during a crisis of pheochromocytoma. Jpn Circ J 1994;58:737-742.

10 Ito K, Sugihara H, Katoh S, Azuma A, Nakagawa M: Assessment of Takotsubo (ampulla) cardiomyopathy using 99mTc-tetrofosmin myocardial SPECT - comparison with acute coronary syndrome. Ann Nucl Med 2003;17:115-122.

11 Bartynski WS: Posterior reversible encephalopathy syndrome, part 1: fundamental imaging and clinical features. AJNR Am J Neuroradiol 2008;29:1036-1042.

12 Granata G, Greco A, Iannella G, Granata M, Manno A, Savastano E, Magliulo G: Posterior reversible encephalopathy syndrome - insight into pathogenesis, clinical variants and treatment approaches. Autoimmun Rev 2015;14:830-836.

13 Lyon AR, Rees PS, Prasad S, Poole-Wilson PA, Harding SE: Stress (Takotsubo) cardiomyopathy - a nove pathophysiological hypothesis to explain catecholamine-induced acute myocardial stunning. Nat Clin Pract Cardiovasc Med 2008;5:22-29.

-14 von Blotzheim LG, Christen S, Wieser S, Ulrich S, Huber LC: Evidence for an association between takotsubo cardiomyopathy and bronchial asthma: retrospective analysis in a primary care hospital. Open Cardiovasc Med J 2015;9:1.

15 Kurahashi H, Okumura A, Koide T, Ando Y, Hirata H, Magota M, Watabane K: Posterior reversible encephalopathy syndrome in a child with bronchial asthma. Brain Dev 2006;28:544-546. 


\section{Case Reports in Neurology}
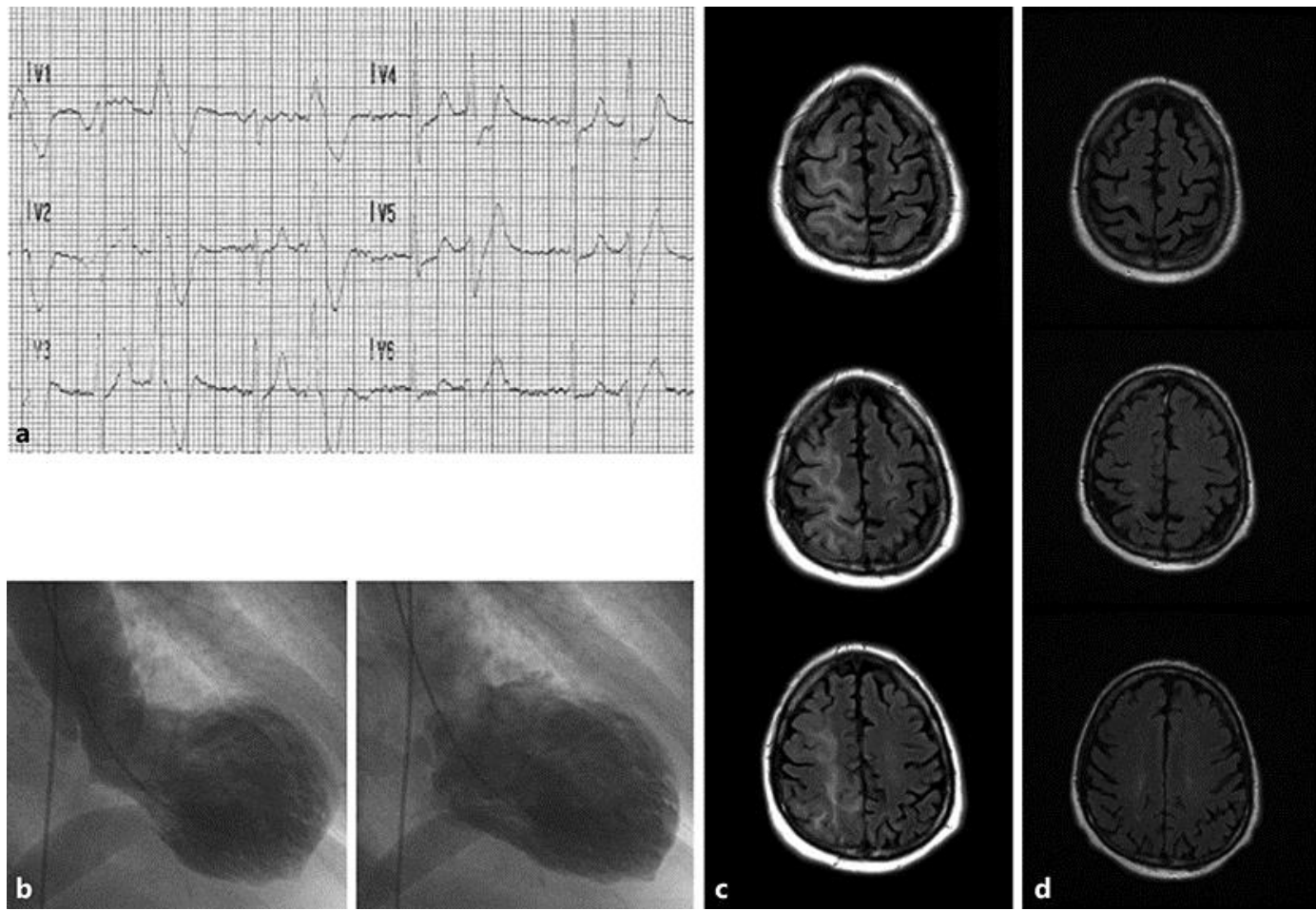

Fig. 1. a ECG with left bundle branch block. b Left ventriculogram with typical stress cardiomyopathy wall motion abnormalities characterized by basal hyperkinesis and apical ballooning. c Brain MRI at day 3: T2 FLAIR. Bilateral, cortical, and subcortical (frontoparietal) vasogenic edema with a right posterior predominance. $\mathbf{d}$ Brain MRI at day 15: T2 FLAIR. No abnormality. 